\title{
REFLEXIONES
}

\section{DEVENIR COMO PSICOTERAPEUTA: UN DIÁLOGO CON EL PENSAMIENTO DE STEPHEN MITCHELL ${ }^{1}$}

\section{N. Montserrat Gómez García²}

Stephen A. Mitchell, considerado el padre del psicoanálisis relacional, no pudo desarrollar desde cero la visión paradigmática de la psicología de dos personas desde el psicoanálisis si no fuera por sus experiencias previas, el contexto socio-cultural en el que se desenvolvió, sin haber leído a los clásicos del psicoanálisis y sin haberse formado en el William Alanson White Psychoanalytic Institute. Considerando que en su época el acceso a la formación de analistas aún era recelado en ciertos institutos para acceso exclusivo a personas con formación médica (Drescher, 2002), en el instituto ya mencionado logra acceder con su grado de psicólogo. No cabe duda de que su pensamiento, actitudes, experiencia y reflexiones tanto conceptuales, técnicas y clínicas fueron formándose gracias a su experiencia vital. En sí, él realizó el recorrido que realizamos todos para ser psicoterapeutas, en el cual somos atravesados por " $n$ " número de elementos vitales que nos marcan para crear nuestro propio estilo como terapeutas.

Reflexiones personales: ¿cómo me veo y me pienso desde el lugar del analista/terapeuta?

Desde el momento de la elección de la profesión de psicólogo hasta el momento de elegir como rama la psicoterapia psicoanalítica van surgiendo dudas sobre las habilidades de uno como clínico y como agente de cambio ante otro que llega a pedir nuestra ayuda. En el recorrido leemos a los autores clásicos, aprendemos sobre las diferentes técnicas y teorías disponibles y paulatinamente vamos desarrollando un criterio de lo que va resonando con nuestro propio estilo personal, pero para llegar finalmente a ese lugar de integración y de criterio original se deben de atravesar por distintas experiencias y aferrarse a alguna base que nos sirva de plantilla. Este recorrido ya lo comenta Mitchell en sus reflexiones sobre su camino psicoanalítico y muchas de sus grandes ideas las atribuye a sus antecesores y es gracias a la historia y a sus experiencias que en su concepción teórica surgió el termino relacional que, junto con Jay Greenberg, supone el puente entre las relaciones interpersonales y la relación de

\footnotetext{
${ }^{1}$ Este trabajo mereció el Primer Accesit en el V Certamen (2015) "Devenir Psicoterapeuta" convocado por el Instituto de Psicoterapia Relacional (Madrid).

${ }^{2}$ Contacto: montserratgomezı3@gmail.com
} 
objetos internos (Mitchell, 2004) por lo que para él el psicoanálisis no es sólo una experiencia unidireccional que se experimenta sólo por el paciente, sino que también es una oportunidad experiencial para el analista (Mitchell, 2015). ¿Qué quiere decir esto y cuál es su planteamiento sobre las relaciones internas y externas entre los objetos en función a la interacción? Lo que hace magnífico su pensamiento es que él logró encontrar un balance entre la teoría de los sistemas intersubjetivos y la de las relaciones objetales para utilizarlas como herramientas con el fin de comprender los mecanismos que se ponen en marcha entre el analista y el analizando en base a un interjuego de interacciones y en el despertar de vínculos, tanto internos como externos, que irán moldeando no sólo la experiencia del paciente hacia una nueva visión de sus conflictos, sino también de lo que el analista va sintiendo y experimentando con cada uno de sus casos y cómo estos le ayudan al clínico a evolucionar también su pensamiento y sus estrategias. Ya decía Mitchell (2015) que el ideal del analista genérico vuelve invisibles las características personales y la dinámica del analista, porque el juicio de cada analista está formado por sus particularidades personales de integración de modelos y concepto psicoanalíticos, así como a sus dinámicas personales, a su carácter y su experiencia vital. No puede haber dos analistas iguales, cada uno tiene su esencia y estilo particular, aunque la base teórica sea la misma o similar.

Por último, lo interesante en su línea de pensamiento es el peso que le atribuye a la autonomía personal, la cual Mitchell (2015) define como algo que se desarrolla a través de la influencia que surge de los procesos de interacción. Tomándolo desde una concepción dirigida hacia lo que es devenir terapeuta, entonces uno va moldeándose gracias a la influencia que vamos recibiendo desde afuera en la interacción con otros que nos van formando un criterio personal en base a lo que nos deja cada vínculo en nuestras reacciones y actitudes en el día a día. Mitchell (2015) mencionaba que la característica esencial de la acción terapéutica del psicoanálisis es el surgimiento de algo nuevo a partir de algo viejo, es decir, que gracias a la experiencia previa y a nuestras interacciones y vivencias presentes podemos crear algo innovador y totalmente nuevo, a pesar de que la ideal principal fuera del pasado. Entonces, la formación como psicoterapeutas se va dando paulatinamente a medida que poco a poco uno va dominando en la formación los distintos conceptos teóricos y va moldeándose en la propia práctica los elementos que nos funcionen mejor para entender a nuestros pacientes y a nosotros mismos en nuestra posición, la cual siempre tendrá un molde base para poder empezar y que poco a poco se irá ajustando hasta que quede perfectamente con nuestro estilo particular.

\section{¿En dónde me coloco como psicoterapeuta?}


Ante la duda de si tenemos las herramientas o el perfil necesario para entrar a la tarea de explorar el material que nos trae un otro que nos demanda ayuda, comprensión, empatía, etc., la actitud que tomemos frente a ese otro, hacia nosotros mismos y hacia nuestra tarea puede ser dificil a veces porque nos damos cuenta que no existen soluciones mágicas ni una pauta genérica que podamos seguir para que el proceso fluya con naturalidad. El psicoterapeuta con poca experiencia se queda atrapado ante la incertidumbre de si realizó la interpretación correcta o de si sus gestos y actitudes son los suficientemente neutrales como para no influir en el tratamiento. Ante esas dudas y miedos, Aron (2003) nos dice que uno debe elegir entre ser uno mismo o ser influenciado por otros cuando entramos en conflicto entre lo que nos han enseñado nuestros supervisores y lo que establece la teoría o lo que nosotros sentimos que es lo correcto en ese momento.

Entonces, a causa de la rigidez a la que nos acostumbramos por querer encontrar una guía práctica y menos dolorosa o intrusiva en el tratamiento, ese conflicto interno entre lo que nos nace y lo que dice la teoría que debemos hacer, Mitchell (2015) nos responde al decir que la disciplina no está en los procedimientos, sino en la sensibilidad con la que el analista participa y gracias a esa sensibilidad tan única que tenemos es como se logrará obtener varios tipos de mente analítica que nos permitirá ser flexibles ante el flujo de diferentes interacciones y dinámicas tan únicas con cada paciente.

\section{El proceso de análisis: ¿Cómo se vive la interacción?}

Un poco menos teórico y más personal, la experiencia subjetiva de cada psicoterapeuta en formación es única e irrepetible, sin embargo, todos partimos de las mismas dudas e inquietudes sobre el desarrollo que tomaremos en la formación y en la práctica clínica. Mitchell (2004) expresaba que su recorrido psicoanalítico fue uno con pocas sorpresas pero con una profunda y enriquecida continuidad y progreso. Creo que con ello quería decir que en este campo uno evoluciona muy dentro de sí porque todo el tiempo estamos en contacto con nuestra racionalización, nuestras emociones y experiencias que van gradualmente madurando y cambiando en nuestras constantes interacciones profundas con otro que también nos siente y que se relaciona con nosotros. Él nos comparte un pedazo de su vida como joven psicoterapeuta al comentar que su supervisora favorita, Geneva Goodrich, le decía que toma alrededor de siete a ocho años el aprender a hacer psicoanálisis, lo cual en su momento fueron palabras que disminuyeron la presión que se daba a sí mismo (Mitchell, 2004). Es quizá en los primeros años de formación en donde se presenta en el psicoterapeuta en formación la mayor presión por sobresalir y por ser el psicoterapeuta más empático y neutral que pudiera existir, pero al vivir en la clínica que es muy complicado e inclusive imposible conseguirlo, que nos 
quemamos la cabeza en nuestro intento por querer dominar desde el principio este arte, es ahí donde me encuentro con Mitchell con las palabras de su supervisora que le calma y lo aterriza a que con la práctica y paciencia logrará con el tiempo aprender a maniobrar este arte, que no trate de comerse tan rápido el mundo.

Así fue como él comprendió que el psicoanálisis era una profesión muy personal y que el rendirnos a la sensibilidad de nuestros supervisores y maestros es la mejor manera para aprender a profundidad lo que ellos tienen que ofrecer (Mitchell, 2004). Uno deja de preocuparse después de eso, al dejarse moldear al principio por los de mayor experiencia en lo que aprendemos a movernos en el arte del psicoanálisis y ya que uno aprenda a entenderlo y a dominar lo básico, entonces uno puede empezar a desarrollar y manifestar su propio arte sin presión, sin querer sacarlo desde el principio sin la base para hacerlo.

\section{Reflexiones sobre la interacción analista-paciente}

Después de un recorrido formativo vasto y de experiencia, se replantea la cuestión de la relación entre analista y paciente, ¿Cómo debe ser? ¿El analista debe ser frío o cálido? ¿Cómo debe ser un analista auténtico? Aron (2003) dice que Mitchell entendió lo intrapsíquico como algo constituido por lo interpersonal y que lo interpersonal es una expresión de lo intrapsíquico, por lo que la mayoría de los analizandos necesitaban sentir que su propio estilo es apreciado para poder abrirse a expandir su repertorio. Entonces tenemos a un analista que en la relación con el paciente proporciona experiencias emocionales correctivas que los transforman a ambos (Mitchell, 2015) ya que en la interacción entre las dos subjetividades se crea un tercer camino que es trazado por los miembros de la díada analítica. Sin embargo, queda el miedo en el analista de mantener la neutralidad de alguna manera y no influir en las decisiones del paciente, el ser un guía suficientemente bueno para que el analizando no pierda el rumbo ni su autonomía. La solución que encuentra Mitchell (2015) para que se salvaguarde la autonomía es que como analistas no neguemos el impacto personal que tenemos frente al otro y reconocer la naturaleza interactiva del proceso analítico. Si bien la metodología del psicoanálisis relacional consiste en que la experiencia subjetiva del paciente es abordada, por lo menos al principio con mucha empatía, a la vez que el analista también realiza su introspección acerca de su propio repertorio teórico y experiencial en su intento por comprender la experiencia del paciente y sus propias limitaciones y entendimientos hacia éste (Ringstrom, 2010) nos topamos con lo que Mitchell (2015) definió como el bootstrapping problem, en el cual uno intenta apoyarse sobre su propio peso para levantarse a sí mismo pero para ello debería de haber algo más, algún punto de apoyo escondido y ese punto de apoyo es la alianza de trabajo. 
Sin una buena alianza de trabajo no podemos hacer nada por levantarnos a nosotros mismos ni a nuestro paciente para que avance el proceso, ambos quedaríamos estancados en un impasse. Por ello, lo que nos enseña la experiencia de Mitchell es que mientras aguantemos, seamos sinceros con nosotros mismos y con nuestro pacientes, no tengamos miedo a lo que surja durante la interacción y tengamos la creatividad y flexibilidad suficiente para escuchar desde otros lugares, nuestros tratamientos y nuestro proceso como psicoterapeutas en formación debería volverse más coherente, interesante y profundo por la experiencia que nos irá moldeando y la introspección que hagamos en el camino para evolucionar y manifestar nuestro propio arte con la ayuda de las bases que hemos recibido durante la formación. Lo que podemos encontrar al leer a Mitchell es que su voz es la de un maestro que logra comprender lo que te está sucediendo y lo que te está pasando en tu recorrido psicoanalítico, te acompaña en el trayecto al mostrarte que nadie puede dominar este arte en el primer intento y que lo esencial para sobrevivir el viaje es relajarse pero sin dejar de mantenernos alerta y siendo verdaderamente auténticos con nosotros, con el proceso y con nuestros pacientes.

\section{REFERENCIAS}

Aron, L. (2003). Clinical Outbursts and Theoretical Breakthroughs A Unifying Theme in the Work of Stephen A. Mitchell. Psychoanalytic Dialogues, 13(2), 273.

Drescher, J. (2002). In Memory of Stephen A. Mitchell, Ph.D. Studies in Gender \&Sexuality.

Mitchell, S. A. (2004). My Psychoanalytic Journey. Psychoanalytic Inquiry, 24(4), 531-541.

Mitchell, S. A. (2015). Influencia y autonomía en psicoanálisis. Madrid, España: Ágora Relacional.

Ringstrom, P. A. (2010). Meeting Mitchell's Challenge: A Comparison of Relational Psychoanalysis and Intersubjective Systems Theory. Psychoanalytic Dialogues, 20(2), 196218.

Original recibido con fecha: 15/01/2016 Revisado: 25/02/2016 Aceptado: 28/02/2016 
Resumen:

¿Cuál es mi lugar como terapeuta? ¿Cómo se siente estar del otro lado del diván? ¿Qué tipo de terapeuta seré? Estas son las preguntas que me he hecho desde que me gradué de la universidad y cuando comencé el máster en psicoterapia psicoanalítica relacional. Creo que son dudas que pueden surgirle a cualquier persona que no se siente aún con las herramientas necesarias para llevar a cabo un proceso de análisis con otro ser humano que pondrá su confianza en ti. Mi analista solía decirme que todas mis preocupaciones acerca de mi devenir como terapeuta son muy válidas y que le preocuparía que no las tuviera. Aunque en este ensayo el énfasis se hará sobre el pensamiento de Stephen Mitchell, y lo que creo que es su concepción de lo que es convertirse en un terapeuta, no será raro que ciertas inquietudes mías se manifiesten en este trabajo al estar realizando mi lectura y mi reflexión sobre este autor y la forma en que éste logró sobrepasar las dudas y los obstáculos con los que me enfrento actualmente en esta nueva etapa de mi carrera profesional.

Palabras clave: Relacional; Autonomía Personal; Inmersión en el punto de vista del Otro; Intrapsíquico; Interpersonal; Alianza de trabajo

Where do I stand as a therapist? How does it feel to be on the other side of the couch? What kind of therapist will I become? These are some of the questions I have asked myself since I graduated and began the master's program in relational psychoanalysis. I believe that these doubts can pop into the head of anyone who doesn't feel sufficiently prepared to carry out an analytical process with another human being who is placing their trust in you. My analyst used to tell me that my worries about becoming a therapist are all valid and that she would have been worried if I didn't have them. Although this paper is intended to expand upon Stephen Mitchell's way of thinking and his beliefs on how to become a therapist. It's inevitable that some of my personal queries will manifest themselves in this paper by considering his ideas and the way he managed to overcome his own doubts and challenges that I'm currently dealing with in this new chapter of my professional life.

Key Words: Relational; Personal Autonomy; Bootstrapping problem; Intrapsychic; Interpersonal; Working Alliance.

English Title: My development as psychotherapist. A Dialogue with the Stephen Mitchell' Thought

\section{Cita bibliográfica / Reference citation:}

Gómez García, N. Monserrat (2016). Mi devenir como psicoterapeuta. Un diálogo con el pensamiento de Stephen Mitchell. Clínica e Investigación Relacional, 10 (1): 313-318. [ISSN 1988-2939] [Recuperado de www.ceir.info ] DOI: 10.21110/19882939.2016.100120 